Elsevier required licence: (c) <2018>. This manuscript version is made available under the CC-BY-NC-ND 4.0 license http://creativecommons.org/licenses/by-nc-nd/4.0/

The definitive publisher version is available online at $h$ ttps://doi.org/10.1016/j.istruc.2018.11.002 


\title{
Case Studies in Topological Design and Optimization of Additively Manufactured Cable-nets \\ Daniel Tish $^{\mathrm{a}, \mathrm{b}, *}$, Wes McGee ${ }^{\mathrm{a}}$, Tim Schork ${ }^{\mathrm{b}}$, Geoffrey Thün ${ }^{\mathrm{a}}$, Kathy Velikov ${ }^{\mathrm{a}}$ \\ ${ }^{a}$ University of Michigan, Taubman College of Architecture and Urban Planning, 2000 Bonisteel Blvd., Ann Arbor, MI 48109, USA \\ ${ }^{b}$ University of Technology Sydney, Faculty of Design, Architecture, and Building, 702-730 Harris Street, Broadway, NSW 2007, Australia
}

\begin{abstract}
Advances in additive manufacturing technologies have availed new modes of design and production across the design and engineering disciplines. While lightweight cable-net structures have long captured the imagination of engineers and architects, there has so far been little research on the opportunities afforded by large-scale additive manufacturing of cable-net structures for form-active or performative tensile surfaces. Additive manufacturing opens up the possibility of manufacturing tensile surfaces beyond knitted, woven nets, or mechanically fastened nets, and expands the types of materials that can be used in such systems. This paper discusses research in novel approaches to the topological design and optimization of cable-nets enabled by the additive manufacturing of elastomeric materials. Through three realized case studies, the topological structure is used to determine the formal, behavioral, and performance-based properties of the cable-net system. This is achieved through the reorientation of a standard quad-grid topology, through a material programming method that embeds non-natural threedimensional forms into two-dimensional patterns, and through a new meso-scale density-based topological optimization method.
\end{abstract}

\section{Introduction}

Additive manufacturing is a rapidly evolving field of manufacturing technology whose advancements in hardware and material applications have been occurring in tandem with the development of computational topological optimization methods. These methods aim to minimize the overall weight of a 
structure through the determination of connectivity, shape, and voids (Deaton and Grandhi [1]). While topological optimization methods are well-researched and widely used in structural engineering, their use in the early stages of architectural design is also continually increasing. Simultaneously, the capacities enabled by additive manufacturing have made it more feasible to directly produce the irregular geometries that are typically the result of topological optimization processes (Tam and Mueller [2], Gardiner [3]). Current research on metamaterials in the material sciences have also leveraged additive manufacturing to produce material constructs in which novel mechanical behaviors or other properties not normally attributable to the raw material itself are ingrained into a material through deliberate structuring at the nano- or micro-scale (Lee et al. [4]).

The BESO method, developed by Querin, Steven and Xie [5], and other hard-kill methods of topological optimization translate particularly well to additive manufacturing fabrication methods since the system is defined by voxels that are constrained to either solid or void conditions (Brackett [6]). On the other hand, density-based methods of topological optimization, exemplified by the SIMP method developed separately by Bendsøe [7] and Zhou and Rozvany [8], typically employ a penalization process to drive intermediate material densities towards binary distinctions, but often leave some voxels just shy of fully solid or void definitions. This necessitates additional post-processing before the additive manufacturing stage for single material prints, but density-based methods without penalization have already been employed successfully for multi-material prints capable of producing a continuous range of densities (Michalatos and Payne [9]).

In addition to the research on topological optimization processes, there has been much work done on the impact of topology networks on form-active structures such as cable-net and tensile membrane structures. In cable-net structures, the individual edges clearly define a line of force in the structure and as such the topological network impacts the flow of force between elements and subsequently the form of the structure at a micro and macro level (Otto [10]). The form-finding of these form-active structures is typically undertaken through the method of particle-spring physics simulation such as those developed by 
Kilian and Ochsendorf [11] or Piker [12]. Employing this method, Ahlquist and Menges [13] have demonstrated the effect that topology can have on simple cable-net structures and argue that topology is the primary input in particle-spring systems. Notably for this work, Coulter and Ianakiev printed auxetic silicone structures over an inflatable surface, using the auxetic topology to adapt to the dynamic changes in the surface area of the inflatable [14].

Despite such interest in cable-net and tensile membrane structures, there is little existing research that investigates the opportunities afforded by large-scale robotic additive manufacturing to fabricate highlycustomized cable-net topologies. As the topology of a cable-net structure functions at the structural, formal, and material levels, the opportunities enabled by a shift to additive manufacturing processes are far reaching. As such, there are multiple perspectives through which to address the design and optimization of additively manufactured cable-net topologies. To address this varied field, this paper presents three realized case studies that develop different approaches to topological design as the critical medium for form-active cable-net structures. Through the case studies presented, cable-net topologies are leveraged to produce novel properties for formal, behavioral, and structural considerations, likening it to other metamaterial constructs and expanding the possibilities for cable-net structures.

\section{Case Studies}

\subsection{Infundibuliforms}

The first case study, entitled Infundibuliforms, is a $30 \mathrm{~m}^{2}$ kinetic cable-net spatial installation (McGee et al. 2018 [15]). The aim of this project was the design of a kinetic, spatially-scaled surface capable of large-scale deformation into variable configurations and to explore the formal and operational possibilities of active tensile surfaces. The installation was comprised of a tensile surface that could be dynamically reconfigured between continuous gradients of vaulted and chimney forms, controlled by three cable-robot actuation systems placed within a bounding steel tube structure whose position is also variable and controlled by three servo motors. As the cable-robots are capable of a range of three- 
dimensional motions that could move up to 2.5 meters from the origin point in multiple directions, the team placed considerable effort in the development of a material system to comprise the reconfigurable surface that would allow for such a multiplicity of forms and their wide range of surface areas.
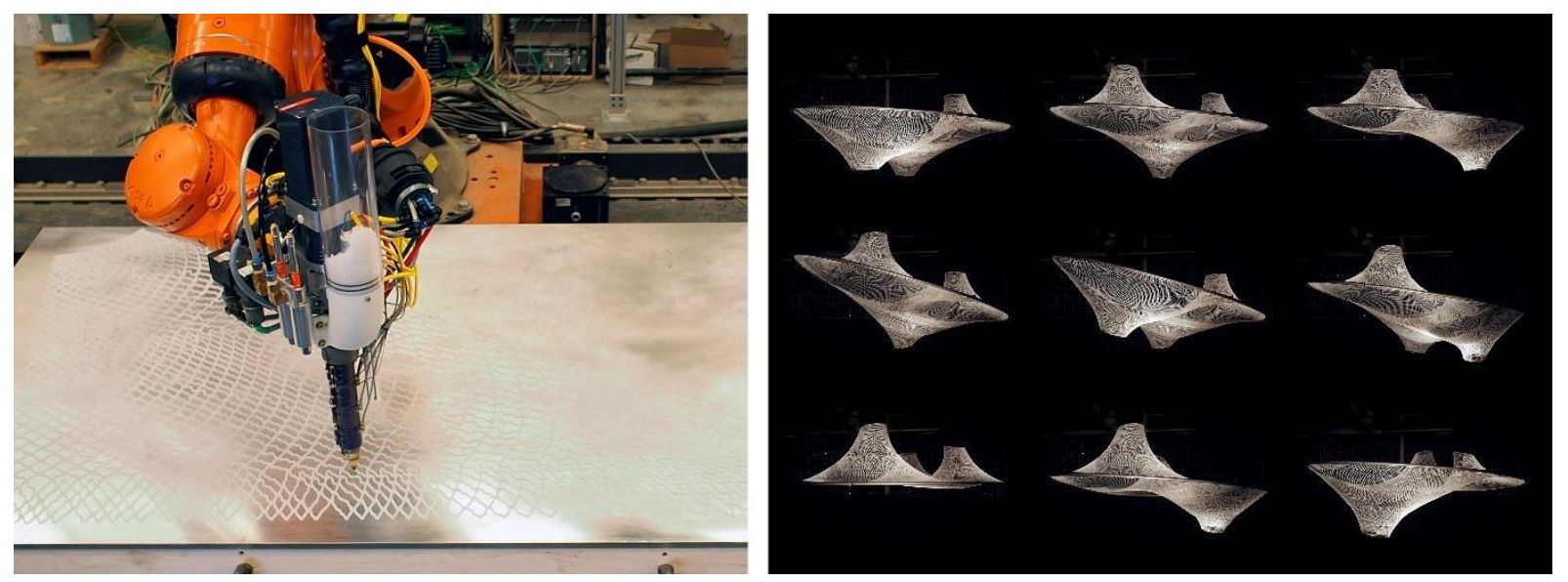

Figure 1: Robotic additive manufacturing work cell and process (L); Nine different positions of the Infundibuliforms installation exhibiting a variety of forms and surface areas while always remaining taut $(\mathrm{R})$.

To address these demands, the authors developed a custom pelletized thermoplastic extrusion end effector for a 6 -axis industrial robot, as well as a $1200 \mathrm{~mm} \times 2400 \mathrm{~mm}$ heated aluminum table that would enable the additive manufacturing process of thermoplastic elastomers (Figure 1, McGee et al. 2017 [16]). This robotic work cell and pellet extruder enabled the production of bespoke elastic cable-nets by allowing the authors to take advantage of the scale and precision of industrial robots in addition to a wider range of thermoplastics than those available in filament form. Thermoplastic elastomers (TPE) are a class of thermoplastics characterized by their high elasticity and surprising strength that have the potential to be recycled and reused. As they are available in a broad range of durometers and melt flows, TPEs were tested across a range of melt flows in an attempt to balance the characteristics of the extruded bead with the ability to produce a void-free crossing joint in the cable net. For this work, an extremely elastic 35A durometer thermoplastic elastomer (TPE) was used for the concentric catenoidal forms, with the reinforcing "saddle" between the concentric zones made of a 68A TPE to provide additional tension in the 
surface to resist self-deflection. Physical tests of extruded TPE elengation under stress provided the inputs for the particle-spring physics simulators used in the design process.

The topological design research was undertaken towards two distinct goals: the tuning of the cable-net topology to guide the active form of the piece towards catenoid, or infundibulum, forms; and the material programming of curving mesh members to embed uniquely tailored three-dimensional forms that diverge from mathematically minimal surfaces in the two-dimensional prints.

\subsubsection{Infundibulum Forming}

In order to explore the effect topology has on the final form, the research leverages particle-spring physics simulators to predict the form of the installation at any given time by solving the interactions between the cable-robot positioning system, the material characteristics of the elastomer, and topological structure of the mesh itself. In particular, the aesthetic or formal goal of the design team was to produce dramatically shaped catenoidal forms at the connection to the cable-robot control rings.

When working with a radial or concentric regular quadrilateral topology, the loaded forms are distinctly conical due to the direct load path from inner ring to outer frame. In order to achieve the anticlastic form through topology, a diagonal grid is introduced by replacing each edge of the original quad grid with a mesh face centered on its midpoint. The diagonal grid exhibits many advantages over the base quad grid, including the desired acutely-shaped anticlastic form due to the spiraling nature of the load paths (Figure 2). The diagonal grid also results in a more uniform structural utilization of the material since both directions of the grid span from inner ring to outer frame, in contrast to the base quad mesh whose radial members take on the majority of the load in the surface. Lastly, the diagonal grid does not result in oddvalence joints at the edges like a quad mesh, where an odd number of edges meet at a vertex and produce a dead-end for the additive manufacturing toolpath. While stop-and-go extrusion procedures are possible, the authors found that the additive manufacturing process is far more robust when the robot doesn't have to make unnecessary starts and stops, and therefore favored a continuous toolpath approach. 

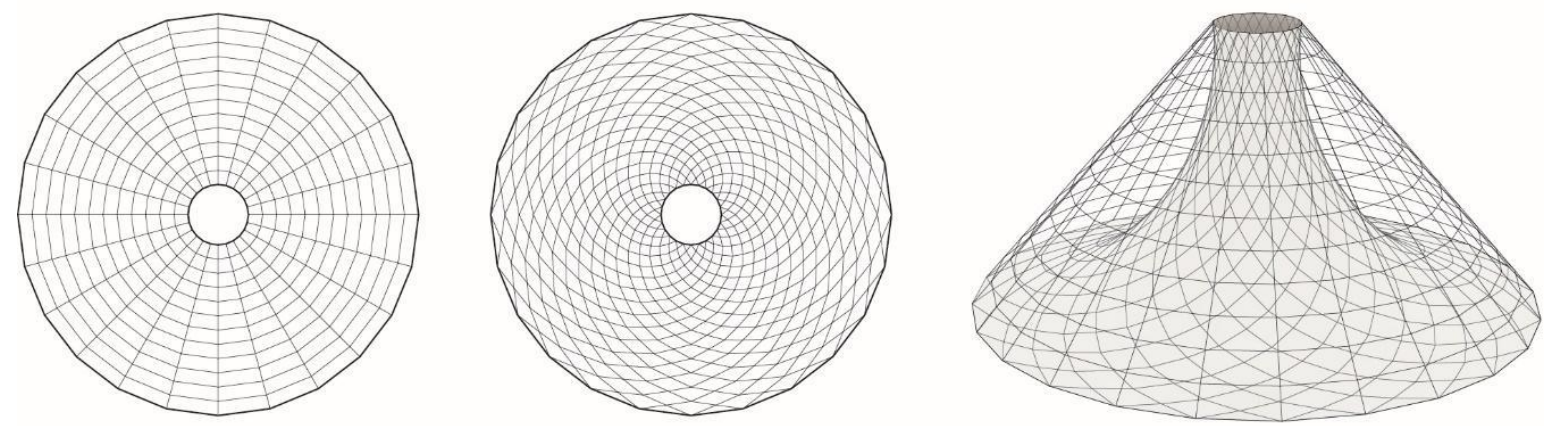

Figure 2: Form-active shapes (R) of regular quad grid (L) and diagonal grid (M) with identical properties as simulated by a particle-spring system

Additionally, a diagonal topology allows the degree of curvature of the catenoid to be tuned across the surface by controlling the subdivisions of the transverse and longitudinal directions, or the $\mathrm{U}$ and $\mathrm{V}$, of the base quad mesh. By increasing the number of concentric subdivisions relative to their radial counterparts, the resultant form-active structure could be more dramatically anticlastic. The final topology of the three funnel-shaped forms utilize this method to increase the curvature of the loaded forms, while also relying on two additional subdivisions in the diagonal grid topology to maintain an appropriate density at the exterior edges and flexibility near the interior ring. As such, the overall form of the prototype installation was largely designed through precise manipulations in the topological structure of the tensile mesh (Figure 3).

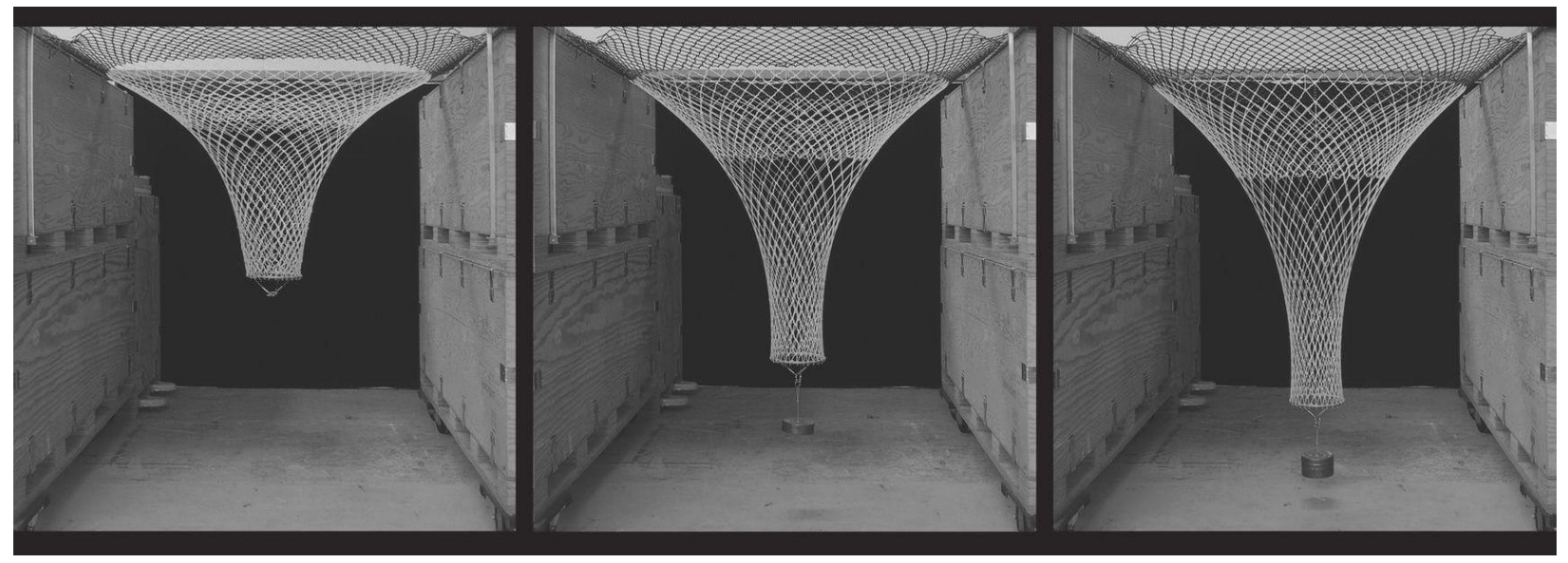


Figure 3: Images of the $1.5 \mathrm{~m} \times 1.5 \mathrm{~m}$ prototype test cable-net made of 35A TPE (white, bottom) and 68A TPE (black, top) exhibiting dramatic anticlastic forms in three different loading conditions.

\subsubsection{Material Programming}

The research also addresses the additional challenge of fabricating custom three-dimensional cable-nets on a two-dimensional surface, so that its formation could only be a result of the combination of topology and material properties. While the thermoplastic material is very elastic and capable of substantial elongations before breaking, the range of the cable-robotic system would have greatly exceeded the elastic capacity of a completely two-dimensional cable-net topology. Therefore, the team set out to find a way to produce the doubly-curved, three-dimensional (3D) cable net structure on the two-dimensional (2D) plane of the heated aluminum surface.

The typical approach to this issue would be to discretize the 3D global surface, reorienting and flattening the parts onto a 2D plane, accepting a minimal displacement in the process. However, this coutureinfluenced approach deals poorly with the changes in surface area that occur while the piece is in motion, leading to an excess of material in the flat state and unsightly self-deflection. As an alternative to this approach, a method of programming the $3 \mathrm{D}$ forms into the $2 \mathrm{D}$ meshes was developed. This material programming is achieved through the concept that the additional edge lengths lost in the projection from $3 \mathrm{D}$ form to $2 \mathrm{D}$ pattern could be reintroduced as curves between the same two endpoints in the $2 \mathrm{D}$ plane. This method leverages the elastic qualities of the particular TPE material in use, for which printed curves easily deform into lines when loaded and return back to their curved state when the load is removed.

This method begins by simply projecting the $3 \mathrm{D}$ form onto the $2 \mathrm{D}$ plane representing its plan figure. The length of the original 3D edges is then compared to the new 2D edges, and the difference between each $3 \mathrm{D}-2 \mathrm{D}$ pair is stored. A particle-spring physics engine is used to iteratively grow the $2 \mathrm{D}$ edges into curves that match the length of their corresponding 3D edge (Figure 4). This is done by subdividing the 2D edges into multiple line segments with free-rotating pin joints connecting them. The physics engine then iteratively increases the rest lengths of line segments, causing the entire edge to curve and buckle. The 
physics engine also incorporates a collision-avoidance system so that as the edges grow and curve, no undesirable intersections between neighboring edges occur and a bead-width minimum distance between edges is maintained for fabrication. Lastly, the intersection angle at each joint in the 3D mesh is also transferred to the 2D mesh due to the fixed nature of the fused joints in the printed cable-net structure.

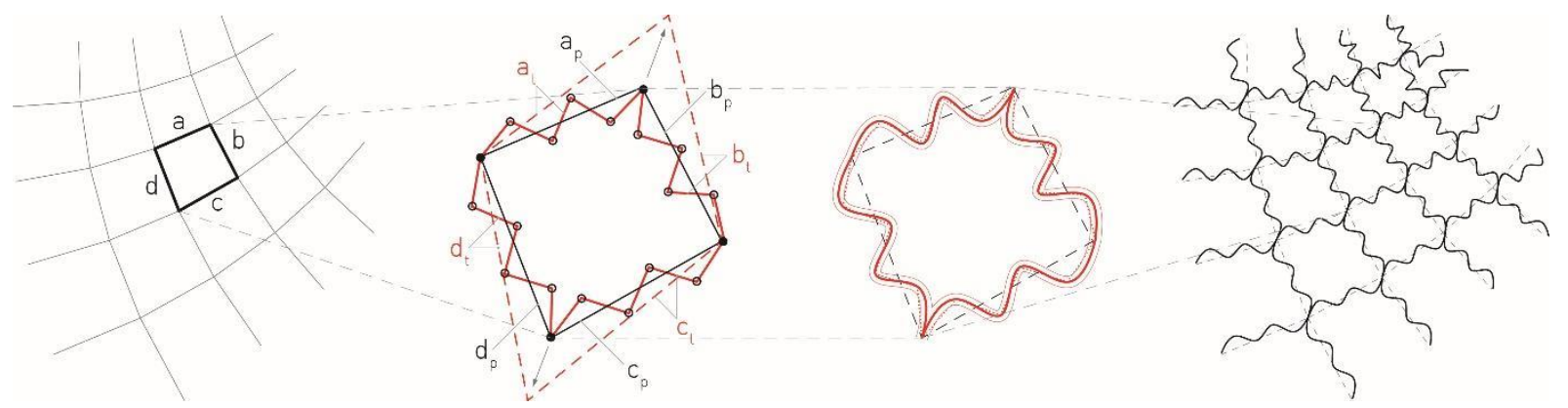

Figure 4: Process of geometric development for programmable geometry of the curving mesh edges.

Since each individual mesh edge is given a custom growth length, this method also avails the opportunity to embed tailored 3D forms that deviate from mathematical minimal surfaces in the 2D topologies. One base 2D pattern could host different 3D forms by embedding curves of different lengths into the original base topology (Figure 5). This potential affects the behavior of the cable-net material system as it alters the way the structure responds to stress and opens it up as a medium for design, similar to a metamaterial construct. The Infundibuliforms installation leverages this ability, as the authors programmed a nonnatural form into the cable-net structure to counteract the rotational bias that would have otherwise been introduced at the cable-robot control rings caused by differing tension forces around the ring.

Most importantly for the Infundibuliforms installation, this method introduces an allowable geometric deformation in addition to the elastic material deformation. This behavior helps negotiate the differences in surface area that the cable-net is subjected to, extending the curves of the topology into lines as the installation nears its extremes and retracting back into the curved state as the rings pass through the center again. This capability not only extends the deformation capacity of the entire structure to take advantage of the range of motion of the cable robot system, but it also helps the surface appear taut in any position that the installation takes. 

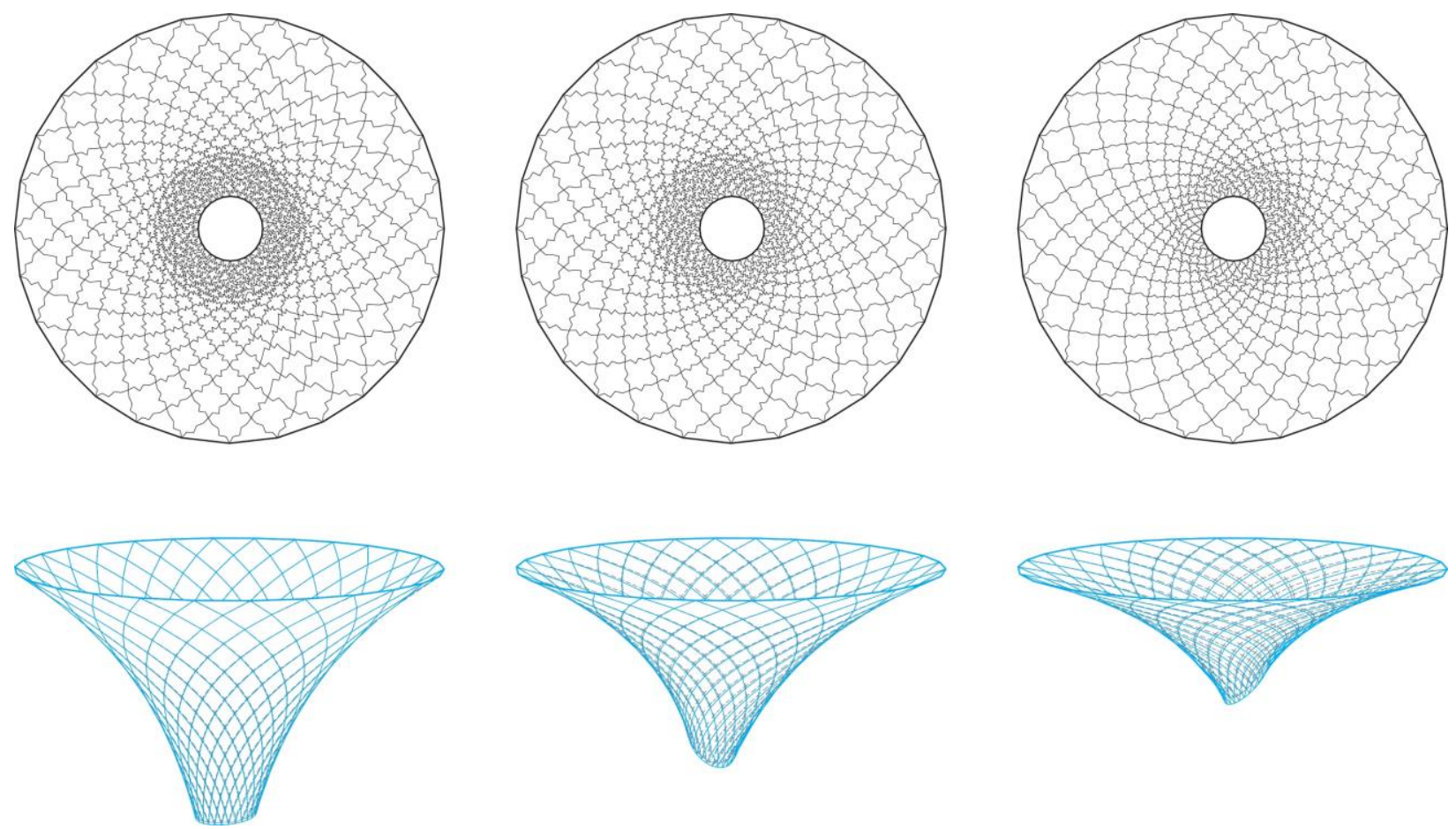

Figure 5: Diagram of three identical two-dimensional cable-nets programmed to match three different threedimensional forms. The resultant programmed topologies in plan (above) and under load (below, in blue) to match their target form (below, dotted gray).

\subsection{Cesca Chair Studies}

In contrast to the use of topological design as a method to influence the final form-active shape, the aim of the Cesca chair case study is to efficiently tune the properties and performance of the additively manufactured cable-net through the topology. Using Marcel Breuer's iconic Cesca Chair as the site for the explorations, these studies reinvent Breuer's woven cane surfaces into continuous, functionally graded cable-net surface. The goal of such explorations is to differentiate the material system in response to the stresses in the surface to efficiently deposit material only where it is needed most, an aim shared by structural topological optimization procedures.

Instead of focusing on the topology optimization at the macro-scale of the cable net, namely the design and specification of each individual load-bearing element, as would be typical in most topological optimization processes, this research develops a method to optimize the meso-scale topology and defines 
metamaterial units with different properties to be aggregated into a complex whole. The interactions between the base material's properties and the anticipated load conditions drive the optimization process which specifies differential densities in the cable net topology to deposit material where it is needed most. In this way, the optimization process is similar to the SIMP method, however without the need for a penalization routine to drive the system towards binary solid or void states due to the ability to print differentiated meso-scale topologies. Thus, rather than presenting a generic optimization approach, the method is enabled by and tailored to the capacities of robotic additive manufacturing, leveraging the ability to create complex, single-bead topologies with high precision to produce metamaterial constructs not possible by other fabrication methods.

\subsubsection{Functionally Graded Topology Construction}

The design process begins by constructing a regular, square-celled mesh of the desired design surface at an appropriately high resolution. Through the use of a particle-spring physics system, the deformation of this base mesh under a typical design load is simulated and a mapping of the strain of each edge member in the surface is produced. This simulated strain data is the basis for the differential nature of the functionally-graded cable net as the optimization sequence attempts to define the material densities for each area in relation to the strain data (Figure 6). This is accomplished by subdividing the design surface into a series of $40 \mathrm{~mm} \times 40 \mathrm{~mm}$ subsurfaces and averaging the strain data of the simulated mesh edges that are contained within each subsurface. The strain data in each subsurface is averaged separately for the U and $\mathrm{V}$ directions in order to introduce anisotropy later in the process if necessary. The average strain data is translated into a new domain defined by a minimum and maximum material density that is determined by the optimization sequence. 

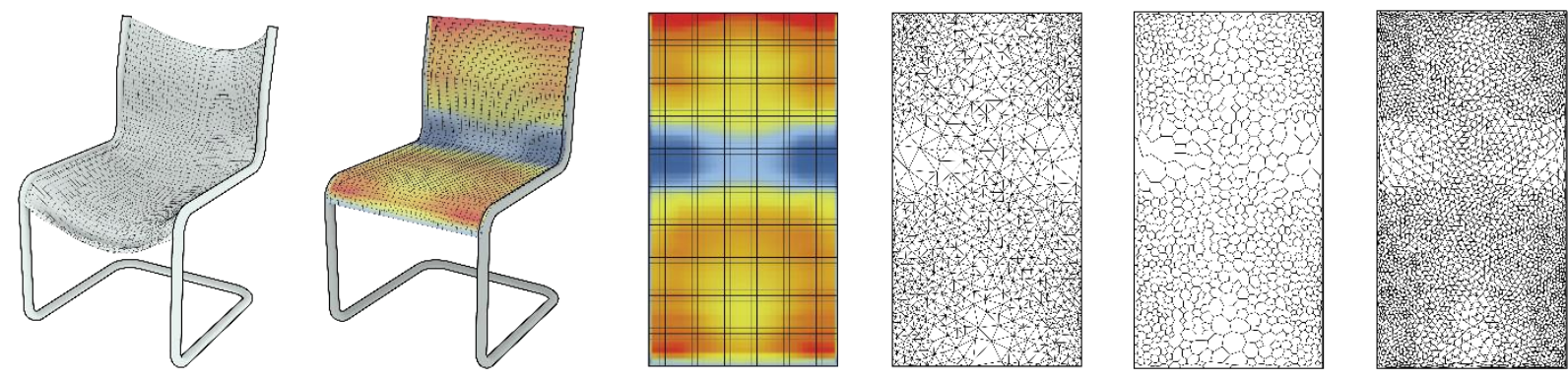

Figure 6: Process diagram for the topological optimization method, from simulated mesh to final toolpath.

New topologies that correspond to the specified material density for each subsurface are generated by either a random uniform distribution method or a subdivision method. The random uniform distribution method distributes a particular number of points randomly in the subsurface and then utilizes Delaunay triangulation to produce the topology, while the subdivision methods uses the transverse and longitudinal strain data to separately specify grid subdivisions of each direction of the surface. At this point, a buffer area sized relative to the local density is introduced between the subsurfaces to blend between the subsurfaces and stitch the topologies back into a cohesive whole.

\subsubsection{Continuous Toolpathing for Robotic Fabrication}

While the outlined method thus far results in cable nets with differential densities, it also produces topologies that contain an uneven valence structure, making it impossible to fabricate them using a singlebead network with one continuous toolpath (Dreifus et al. [17]). Delaunay triangulation regularly produces vertices within the topology that are connected with an odd number of edges, and these conditions constitute dead-ends in robotic additive manufacturing processes. By eliminating odd-valence vertices, the topology can be translated into a single, continuous toolpath. This is achieved by an algorithmic weaving operation within each cell of the dual graph of the differential density mesh. The weaving operation replaces each edge of the dual graph with a vertex at the midpoint, connecting this vertex to two of its neighboring vertices within the cell with new edges. Since each edge, with the 
exception of the exterior edges, is shared between exactly two faces, the result is a topology where every vertex is connected to four edges.

After the algorithmic weaving operation, the new series of unordered edges must be joined into a single continuous toolpath with full cross-over joints at every intersection for superior structural performance to joints in which two segments attempt to meet at a point. This is accomplished using a custom script which first maps the connectivity of each vertex in the topology, creating a list of adjoining edges. Once a starting point is chosen, the script samples the list of edges connected to the endpoint of the current edge and sorts them radially about the current endpoint. Once the adjoining edges are sorted, the edge opposite of the current edge can be quickly found using a modulo function. This cycle repeats until the toolpath closes itself off or all edges have been joined (Figure 7). The radial sorting method performs better than operations which attempt to compare vectors to determine the opposite edge due to the irregularity of the intersections formed in the topological optimization process.
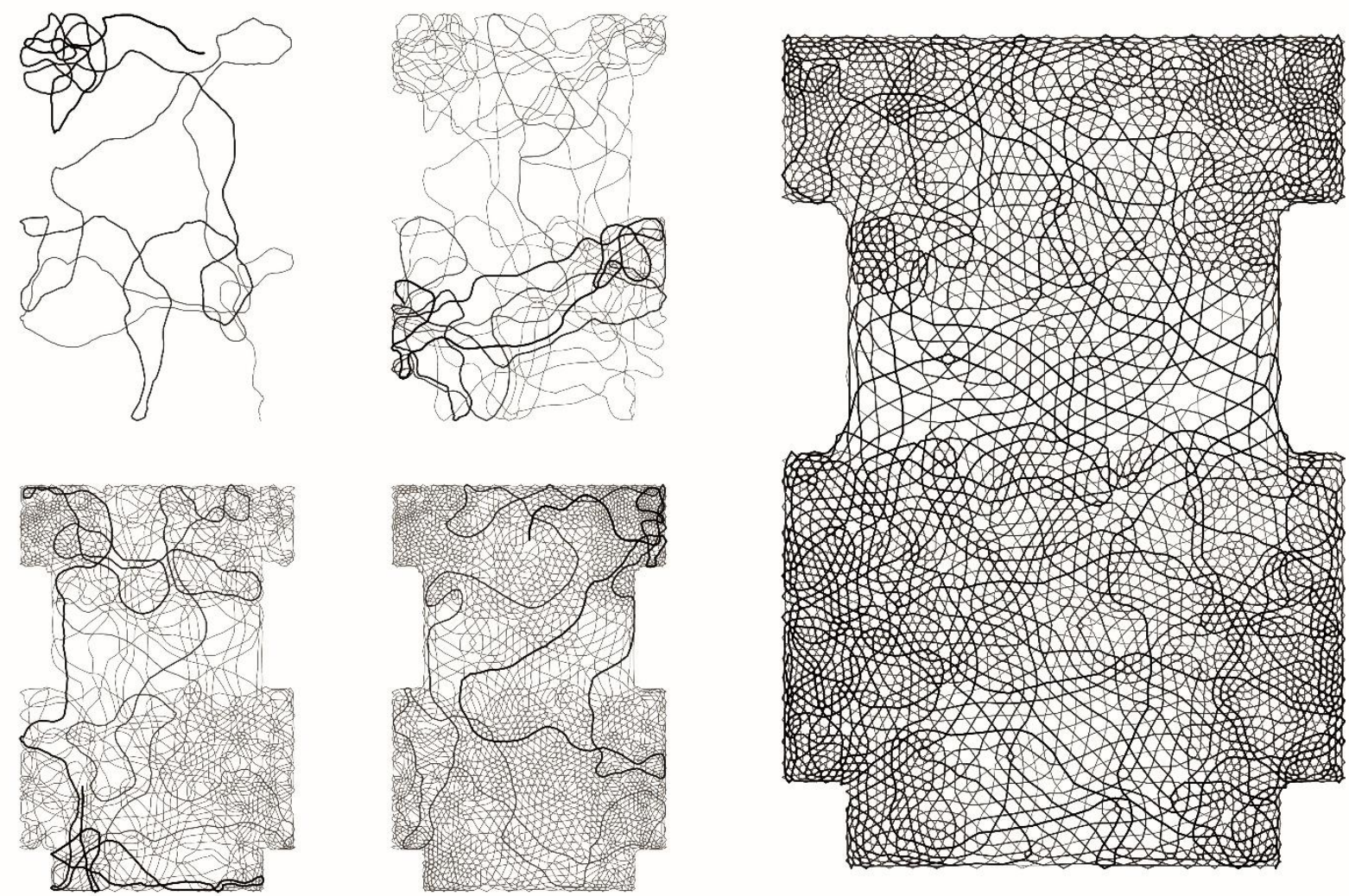
Figure 7: (L) In progress continuous toolpaths after 1000, 4000, 8000, and 12000 edges (L to R, T to B), highlighting the last 1000 edges with progressively thicker and darker lines to illustrate the crossover nature of each intersection. (R) Full toolpath, colored to show the earliest edges printed (light gray) and the latest (black).

\subsubsection{Meso-Topology Optimization}

The optimization process determines the minimum and maximum material densities that will minimize material usage of the continuous toolpath cable net topology while meeting a given performance criteria. This process is achieved through the use of a combination of multi-variable solvers. The algorithmic solvers have control over the parameters that define the bounds of the material density range in addition to two further parameters that can produce nonlinearity in the mapping from strain to density. To establish the fitness of each individual in the optimization process, the maximum deformation of each individual topology is simulated using finite element analysis with the same typical design load that informed the initial strain mapping. The individual's maximum deformation is compared against an allowable deformation threshold, and those individuals that do not meet this criterion are penalized in relation to their difference from the threshold. This deformation score is combined with an estimation of the total mass of the printed topology to produce a single optimization objective to be minimized. Using the NLOpt library [18], a global evolutionary algorithm [19] first searches for a coarsely optimal solution, which is then locally refined through the use of the Subplex algorithm [20]. When the fluctuations in the final fitness criteria have fallen below 0.001 for the typically double-digit scores, the resultant topology is considered optimized (Figure 8). 

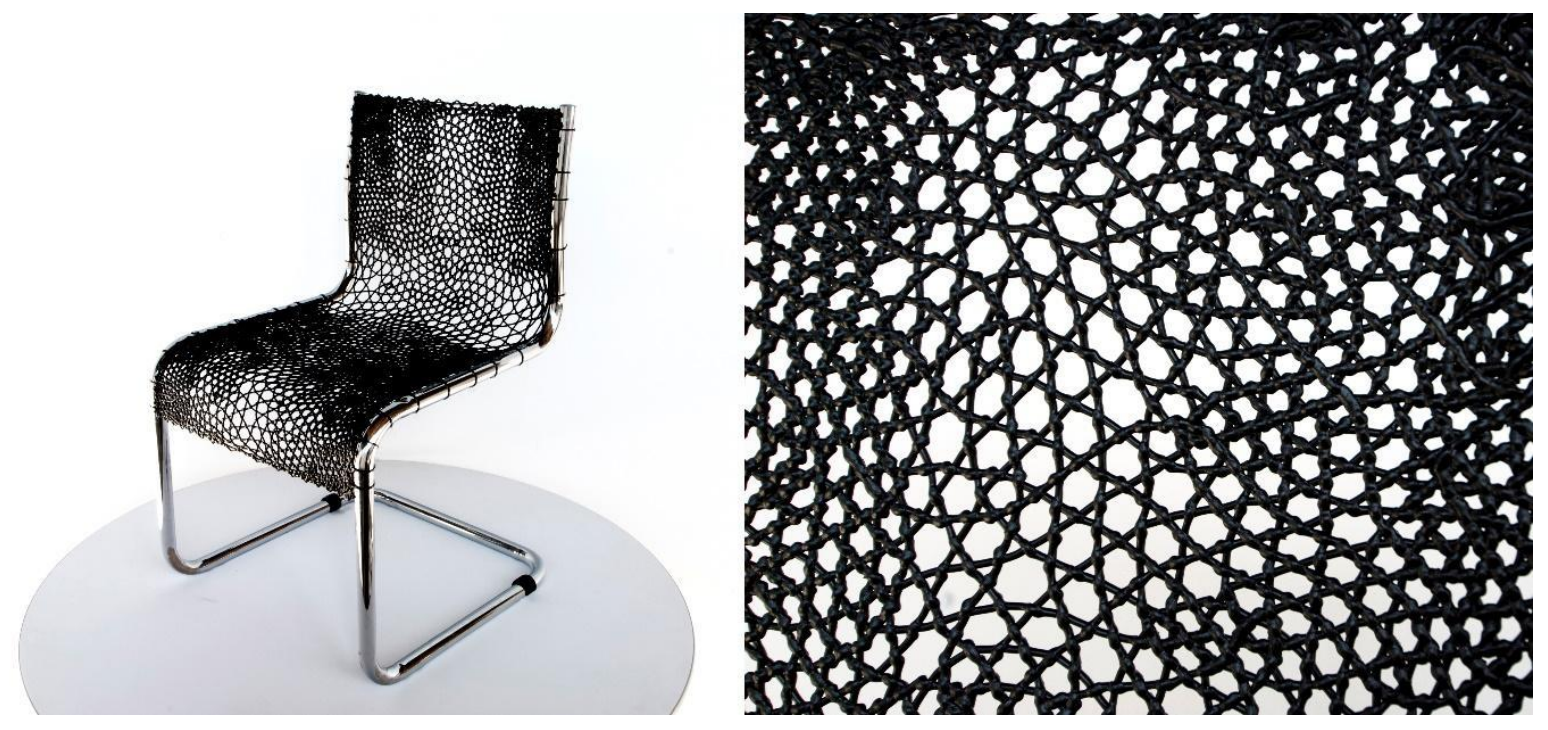

Figure 8: Final result of the random distribution topological optimization process on the Cesca chair (L), detail of the additively manufactured cable-net topology $(\mathrm{R})$.

\subsubsection{Optimization Results}

In addition to the design of the functionally graded surface for the Cesca chair, the research also employed the developed method on a series of test rigs for numerical comparison. The test rig measures $850 \mathrm{~mm} \times 850 \mathrm{~mm}$ and is tested with a number of standardized weights to accurately gauge performance across different printed configurations.

The Cesca chair surface transforms the discrete seat and back of the original design into a continuous surface that follows the contours of the frame from the top of the chair to just below the knee. The optimized topology places additional material adjacent to the frame of the chair, especially in areas near the top of the back and across the seat, while the structure is most sparse in the transition zone between seat and back (Figure 6). The densities of the subsurface areas range from a minimum of $0.815 \mathrm{~kg} / \mathrm{m}^{2}$ to a maximum of $2.61 \mathrm{~kg} / \mathrm{m}^{2}$, where a density of $2.84 \mathrm{~kg} / \mathrm{m}^{2}$ would represent a completely solid cell at the same extrusion height. The algorithmic weaving pattern of the continuous toolpath topology hearkens back to Breuer's woven cane seating surfaces in the original chair design, though conceived and fabricated through $21^{\text {st }}$-century technologies. 
The test rig cable nets are designed to resist the load of a $7.25 \mathrm{~kg}$ weight placed in the center of the surface, and the optimizations were set up to produce a surface that would deform less than $60 \mathrm{~mm}$. An optimized cable net was designed using both the random uniform distribution and subdivision methods to compare the performance of each method. The resultant optimized random uniform distribution net weighed only nominally more than its subdivision counterpart. Additionally, a standard, square-celled cable net with the same overall weight as the average of the two optimized nets was fabricated as a base comparison with the same material usage (Figure 9). The subdivision method result exhibited the best performance by deforming $90 \mathrm{~mm}$ under load, as compared to $97 \mathrm{~mm}$ for the random uniform distribution method net, suggesting that the ability to control the anisotropy and direction of the cable net topology helps deliver greater structural efficiency. The square-celled comparison net deformed $112 \mathrm{~mm}$ under load, which results in an overall increase in performance of $24.4 \%$ for the subdivision method and $15.5 \%$ for the random uniform distribution method. While neither of the cable nets matched the design criteria, this can be attributed to a need for further refinement of the physical material properties within the FEA simulation, as each physical piece exceeded their simulated deformations in nearly equal proportion.
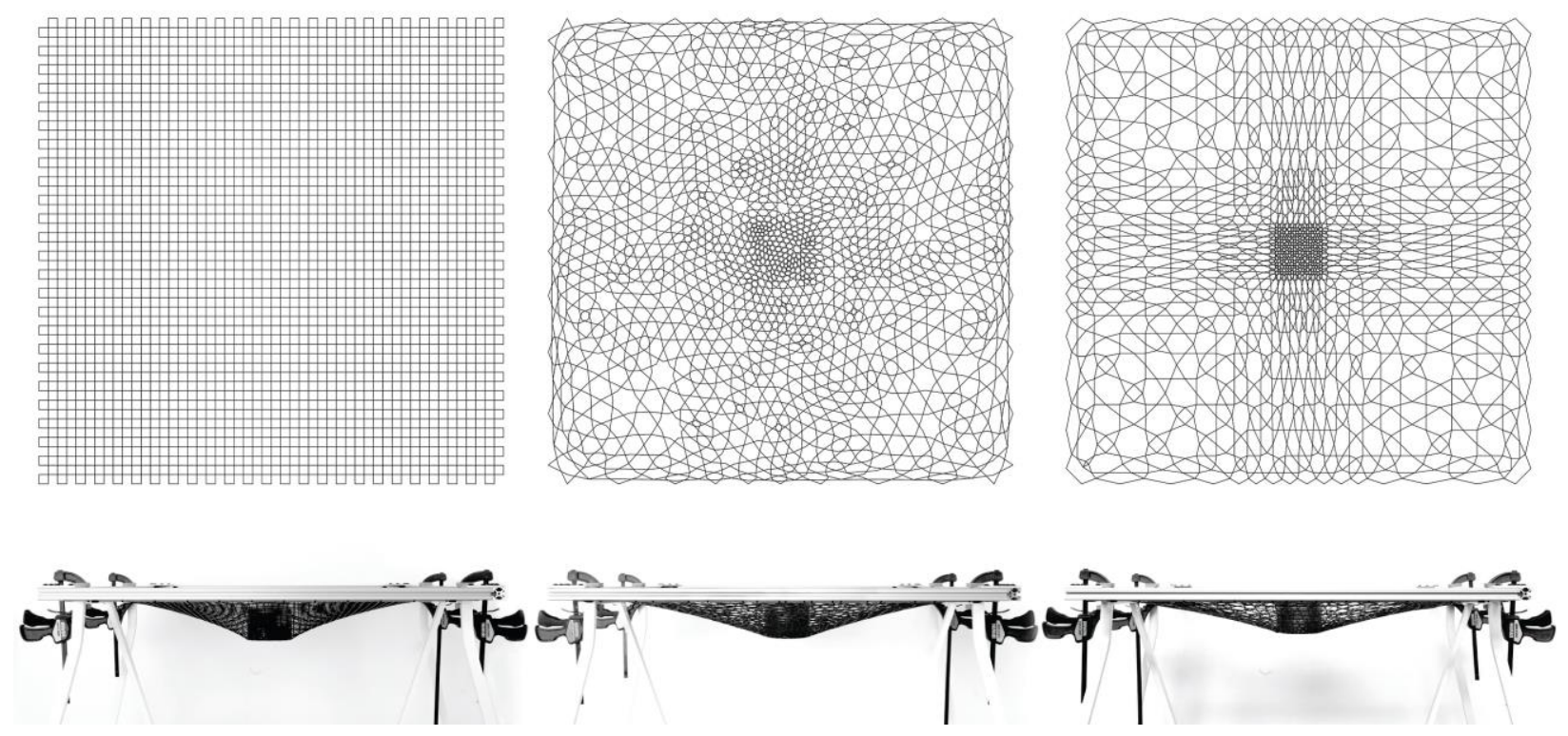
Figure 9: Plan figures and physical deformation results of test rig cable nets: ( $\mathrm{L}$ to $\mathrm{R}$ ) base square-celled, optimized random uniform distribution method, and optimized subdivision method.

\subsection{Transient Geometries}

The Transient Geometries installation at the University of Technology Sydney offered an opportunity to scale up the topological optimization methods developed through the Cesca Studies and to combine these with the programmable meshes from the Infundibuliforms research. The case study also engaged with more complex global topologies in the construction of the form-active structures, specifically those with a 3D base geometry. The case study's form-active shape creates a complex continuous surface spanning from one end of the building lobby to the other, engaging with the lobby column by wrapping down it and anchoring to the ground at its base, and features an actuated funnel form that senses and responds to the pedestrian flow entering and leaving the building (Figure 10). This case study was fabricated using a custom-built filament extruder end effector and a UR10 robot with a $1200 \mathrm{~mm} \times 1200 \mathrm{~mm}$ heat bed. The material in use for this installation was an 85A durometer thermoplastic polyurethane due to the necessity for a stiffer material to feed through the filament extruder.

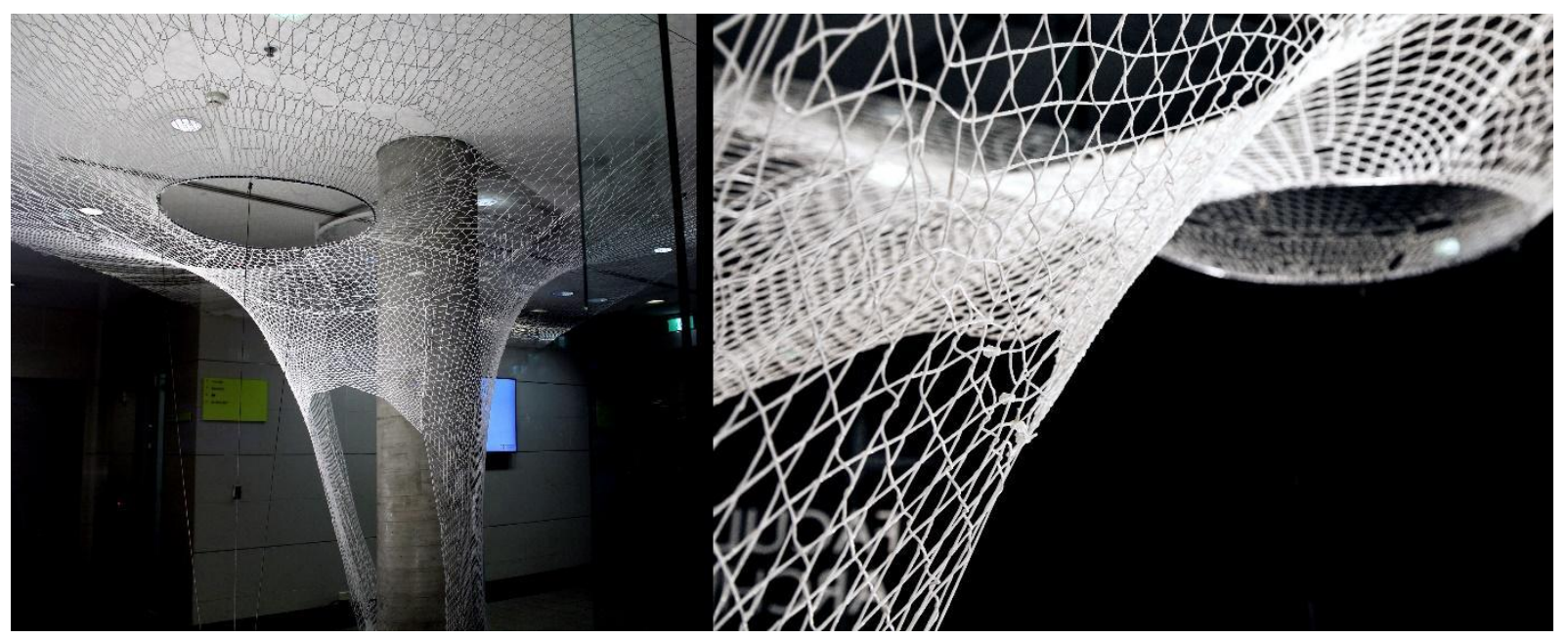

Figure 10: Transient Geometries installation at the University of Technology Sydney.

The topology of the cable-net structure is graded in response to the stresses in the surface using the methods developed by the Cesca Chair studies. The portions of the surface that surround the lobby's 
column and anchors to the ground carry the most tension in the system and are therefore the densest, while the outer areas of the overhead surfaces carry the least stress and are the most open (Figure 11). The actuated funnel form utilizes the material programming methods developed in Infundibuliforms to help adapt to its changing shape. In the process of trying to combine these two methods developed for unique purposes, however, some compatibility issues arose. Since the topological pattern of the graded surfaces is comprised mainly of a series of interconnected triangles, the material programming physics engine struggled to find space to add extra material with such an inflexible topology, as compared to one made of quadrilaterals, and only half of the additional material was able to be programmed in.
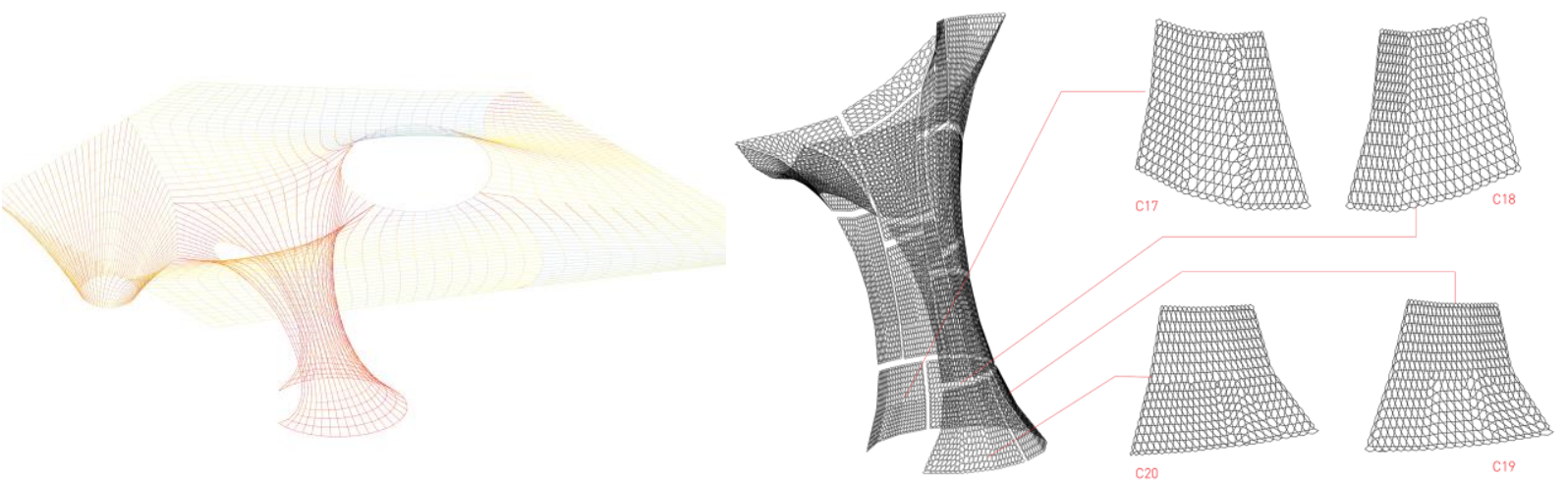

Figure 11: (L) Strain diagram of the entire cable-net surface for the Transient Geometry installation. (R) Detail perspective of functionally graded topology surrounding the in-situ column and individual panel plans.

\section{Discussion}

Taken as a whole, the case studies offer a wide range of frameworks through which to engage the topological design of cable net structures. The initial anticlastic studies of the Infundibuliforms research explore how simple changes to topological structure can radically alter the form-active shape of cable-net structures. Subsequently, by questioning the linear nature of the topology's constituent members and replacing them with programmable curves, the second half of the Infundibuliforms research approaches the design of the material system from a behavioral standpoint, altering how the structure responds to loads and stress. The topological optimization methods developed during the Cesca chair studies create a 
framework to produce differential structural properties within a single material system in order to efficiently place material only where it is needed most. Finally, the Transient Geometries installation looks to combine multiple aspects of these studies at a large scale and with complex base geometries.

This research takes place in a context of diverse applications for these cable net structures, with the dichotomy between the shape-shifting and form-giving nature of the Infundibuliforms research to the load-carrying capacity of the Cesca chair studies laying out two extremes in terms of required performance and behavior. These applications suggest at once new, exciting futures for cable net structures where continuous material gradients provide efficient use of resources for the already lightweight structures. Additionally, new forms of material agency arise through applications in dynamic structures in which the heterogeneous material systems lead to structures that are actively shaped through a conversation between user, environment, and material.

As the research so far has engaged with cable-net structures on a macro- and meso-scale, the next steps of the research will investigate how the micro-scale could be an arena for design through multi-material additive manufacturing. The ability to deposit materials of different strengths or durometers across the structure would alter the requirements for the topology and the behavior of the final form. A custom dualfeed progressive-cavity extruder end effector for the industrial robot has been recently built and preliminary material and control tests are currently underway. This new end effector deposits a wide range of materials including thermoset plastics and silicones and can be controlled to produce gradients between the two material feeds. These capabilities offer greater control over the materials that comprise the cable-net and produce the ability to alter the structural or behavioral properties of the structure separate from or in conjunction with the topological structure.

\section{Conclusion}

As the presented case studies demonstrate through both topological design and topological optimization, the design of additively manufactured cable-net structures produces a dramatic reversal of the typical form-material relationship, and the reorientation from the formal towards the material domain signals an 
engagement with morphogenetic modes of design. Rather than the normative mode in which a form is designed and a material is forced to conform to its specified shape, here the material system is designed in such a way that the desired formal qualities or performance characteristics are brought about through material agency in a bottom-up approach.

Robotic additive manufacturing techniques have the ability to change the way that designers not only fabricate but conceive of and design cable-net structures. Custom topological organizations which would have been difficult to accurately achieve by prior manual or mechanical methods, can now be designed in standard CAD environments and fabricated easily. This ability opens the topological structure, long known to be a key factor to the form-active shape of cable-nets, to new modes of design, optimization, and material application. This paper has presented three such novel approaches to topological design and optimization, conceiving of it as a part critical part of the metamaterial construct, and developing ways to program variations in form, behavior, and performance into the topology itself.

\section{Acknowledgements}

Infundibuliforms was funded by the Taubman College of Architecture and Urban Planning 2016 Research Through Making Grant and the University of Michigan Office of Research Small Projects Grant. It was installed in the Taubman College Liberty Research Annex Gallery in March and October of 2016. Fabrication Assistants for this project were: Dustin Brugmann, Asa Peller, Andrew Kremers, Andrew Wald, Iram Moreno Pinon, and Isabelle Leysens.

The Transient Geometries installation was the final outcome of a two-week intensive summer masterclass at the University of Technology Sydney, taught by Daniel Tish, Tim Schork, and Tran Dang, and in collaboration with Teresa Vidal Calleja, Gavin Paul and Sheila Sutjipto from the UTS Centre for Autonomous Systems. The authors want to thank the following student participants for their work: Dylan Astawa, Beau Avedissian, Carol Huang, Celina Lui, Nariya Rahman, Timothy Vornfett, Emon Saleh, Roy Liu, James Graham, Yiran Dong, Karina Alzate, Rainy Huang, and Paul Phan. 


\section{References}

[1] J. D. Deaton and R. V. Grandhi, "A survey of structural and multidisciplinary continuum topology optimization: post 2000," Structural and Multidisciplinary Optimization, vol. 49, no. 1, pp. $1-38,2014$.

[2] K.-M. M. Tam and C. T. Mueller, "Additive Manufacturing Along Principal Stress Lines," 3D Printing and Additive Manufacturing, vol. 4, no. 2, pp. 63-81, 2017.

[3] J. Gardiner, "Exploring the Emerging Design Territory of Construction 3D Printing - Project Led Architectural Research," PhD Thesis, School of Architecture and Design, RMIT, 2011.

[4] J. H. Lee, J. P. Singer, and E. L. Thomas, "Micro- /Nanostructured Mechanical Metamaterials," Advanced Materials, vol. 24, no. 36, pp. 4782-4810, 2012.

[5] O. M. Querin, G. P. Steven, and Y. M. Xie, "Evolutionary structural optimisation (ESO) using a bidirectional algorithm," Engineering Computations, vol. 15, no. 8, pp. 1031-1048, 1998.

[6] D. Brackett, I. Ashcroft, and R. Hague, "Topology optimization for additive manufacturing," in Proceedings of the solid freeform fabrication symposium, Austin, TX, 2011, vol. 1, pp. 348-362.

[7] M. P. Bendsøe, "Optimal shape design as a material distribution problem," Structural optimization, vol. 1, no. 4, pp. 193-202, 1989.

[8] M. Zhou and G. Rozvany, "The COC algorithm, Part II: topological, geometrical and generalized shape optimization," Computer Methods in Applied Mechanics and Engineering, vol. 89, no. 1-3, pp. 309-336, 1991.

[9] P. Michalatos and A. O. Payne, "Working with multi-scale material distributions," in ACADIA 2013 Adaptive Architecture, P. Beesley, M. Stacey, and O. Khan, Eds. Cambridge, Ont.: ACADIA, 2013. 
[10] F. Otto, IL-8: Nets in Nature and Technics (Mitteilungen des Instituts für Leichte Flächentragwerke (IL)). Stuttgart: University of Stuttgart, 1975.

[11] A. Kilian and J. Ochsendorf, "Particle-spring systems for structural form finding," Journal of the International Association for Shell and Spatial Structures, vol. 46, no. 148, p. 77, 2005.

[12] D. Piker, "Kangaroo: form finding with computational physics," Architectural Design, vol. 83, no. 2, pp. 136-137, 2013.

[13] S. Ahlquist and A. Menges, "Realizing Formal and Functional Complexity for Structurally Dynamic Systems in Rapid Computational Means: Computational Methodology based on Particle Systems for Complex Tension-Active Form Generation," in Advances in Architectural Geometry 2010, C. Ceccato, L. Hesselgren, M. Pauly, H. Pottmann, and J. Wallner, Eds. Vienna: SpringerVerlag, 2010.

[14] F. B. Coulter and A. Ianakiev, "4D Printing Inflatable Silicone Structures." 3D Printing and Additive Manufacturing, vol. 2, no. 3, pp. 140-144, 2015.

[15] W. McGee, K. Velikov, G. Thün, and D. Tish, "Prologue for a Robotic Architecture: Bridging design environments, robotic additive manufacturing, and systemic controls for kinetic in-formation," in Towards a robotic architecture, M. Daas and A. J. Wit, Eds. New York: ORO Editions, 2018.

[16] W. McGee, K. Velikov, G. Thun, and D. Tish, "Infundibuliforms: Kinetic Systems, Additive Manufacturing for Cable Nets, and Tensile Surface Control," in Fabricate 2017: Rethinking design and construction, B. Sheil, A. Menges, R. Glynn, and M. Skavara, Eds. London: UCL Press, 2017, pp. 84-91.

[17] G. Dreifus, K. Goodrick, S. Giles, M. Patel, R. M. Foster, C. Williams, J. Lindahl, B. Post, A. Roschli, and L. Love, "Path Optimization Along Lattices in Additive Manufacturing Using the Chinese Postman Problem." 3D Printing and Additive Manufacturing, vol. 4, no. 2, pp. 98-104, 2017. 
[18] S.G. Johnson, The NLopt nonlinear-optimization package, http://ab-initio.mit.edu/nlopt.

[19] C. H. da Silva Santos, M. S. Gonçalves, and H. E. Hernández-Figueroa, "Designing Novel Photonic Devices by Bio-Inspired Computing," IEEE Photonics Technology Letters, vol. 22, no. 15, p. $1177,2010$.

[20] T. H. Rowan, "Functional stability analysis of numerical algorithms," Ph.D. Thesis, Department of Computer Sciences, University of Texas at Austin, 1990. 